17

\title{
Усиление коротковолнового излучения на основе резистивной неустойчивости релятивистского электронного потока (квазиоптическая теория)
}

\author{
(C) Н.С. Гинзбург, А.М. Малкин, И.В. Железнов, А.С. Сергеев, Е.Р. Кочаровская
}

Институт прикладной фризики РАН, 603950 Нижний Новгород, Россия

e-mail: ginzburg@appl.sci-nnov.ru

(Поступило в Редакцию 25 ноября 2016 г.)

В рамках квазиоптического подхода исследованы линейная и нелинейная стадии неустойчивости электронного потока, движущегося вблизи плоской границы поглощающей среды, диссипативные характеристики которой описываются импедансными граничными условиями Леонтовича. Показано, что резистивная неустойчивость может быть использована для усиления излучения коротковолновых диапазонов, включая терагерцовый. Определены инкременты неустойчивости и эффективность энергообмена.

DOI: 10.21883/JTF.2017.08.44732.2111

\section{Введение}

Резистивная (диссипативная) неустойчивость, возникающая при движении электронных пучков вблизи металлических поверхностей с конечной проводимостью, хорошо известна как в электронике СВЧ, так и в физике ускорителей [1-4]. В рамках линейного гидродинамического описания она представляет собой канонический пример возбуждения волн с отрицательной энергией [1]. Паразитная модуляция плотности пучка и потери энергии электронов, связанные с данной неустойчивостью, являются важными факторами, которые учитываются при проектировании и работе ускорителей [2]. С другой стороны, известны примеры использования резистивной неустойчивости для усиления волн сантиметрового диапазона [4]. В ряде работ рассматривалась возможность использования этого эффекта для генерации излучения оптического диапазона [5].

В частности, в работе [4] теория резистивной неустойчивости строилась применительно к клистронной схеме усилителя сантиметрового диапазона, где подобный механизм используется для повышения эффективности группировки частиц в области дрейфа. При этом предполагалось, что частота усиливаемого излучения меньше критической частоты низшей моды формирующего пространство дрейфа волновода, стенки которого покрыты поглощающим материалом.

В настоящей работе в проекции на коротковолновые диапазоны исследуется противоположный предельный случай, когда резистивная неустойчивость развивается при прямолинейном движении ленточного электронного пучка над поглощающей поверхностью в отсутствие волноведущих систем (на практике - в сверхразмерном планарном волноводе, когда вторая удаленная от пучка параллельная пластина не оказывает влияния на процесс взаимодействия). В этом случае поля излучения в отличие от [4] представляются в виде распространяющихся волн и характеризуются конечным значением продольной и поперечной компонент вектора Пойнтинга. Ниже приводится анализ резистивной неустойчивости в рамках квазиоптического приближения, в котором распространение излучения над поглощающей поверхностью описывается параболическим уравнением. Данный подход позволяет рассмотреть как линейную, так и нелинейную стадии развития неустойчивости.

Следует отметить, что в отсутствие электронного пучка использованное в работе квазиоптическое приближение восходит к классической работе [6], где на его основе был проведен анализ распространения поверхностных волн Ценнека-Зоммерфельда. Однако такие волны не являются замедленными и поэтому не могут быть возбуждены посредством взаимодействия с прямолинейным электронным пучком. Развитие диссипативной неустойчивости в исследуемой системе обусловлено оттоком энергии из имеющей отрицательный знак энергии медленной волны пространственного заряда.

Работа имеет следующую структуру. В разд. 1 в рамках квазиоптического приближения построена самосогласованная модель, позволяющая описать линейную и нелинейную стадии резистивной неустойчивости. В разд. 2 получено дисперсионное уравнение, на основании которого исследован механизм развития неустойчивости и определены ее инкременты. Раздел 3 посвящен описанию нелинейной стадии, которая позволяет опре-

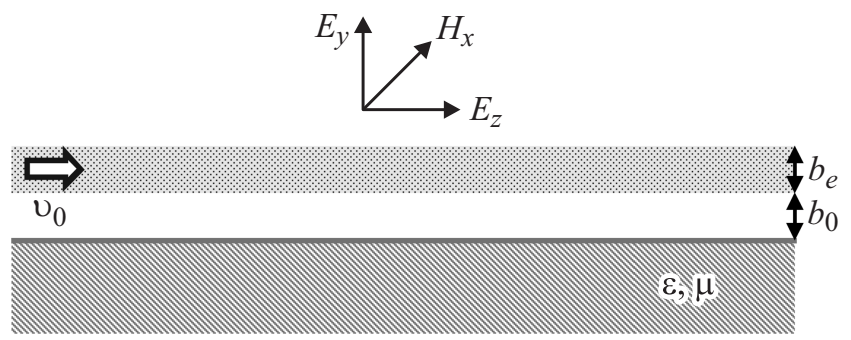

Рис. 1. Двумерная модель резистивного усилителя с прямолинейным релятивистским электронным пучком. 
делить эффективность энергообмена. Проведены оценки возможности усиления терагерцового излучения на основе исследуемого механизма.

\section{1. Модель и основные уравнения}

Пусть ленточный релятивистский электронный поток движется прямолинейно с поступательной скоростью $\vartheta_{0}=\beta_{0} c$ в сильном ведущем магнитном поле, параллельном плоской поверхности раздела „материалвакуум“(рис. 1). Ось $z$ направлена вдоль магнитного поля, ось $y-$ по нормали к поверхности. Рассматривается двумерная модель: система считается бесконечной по третьей координате $x$. Однородный изотропный материал заполняет полупространство $y<0$ и характеризуется комплексной диэлектрической $\varepsilon=\varepsilon^{\prime}-i \varepsilon^{\prime \prime}$ и действительной магнитной $\mu$ проницаемостями, удовлетворяющими условию $|\sqrt{\varepsilon \mu}| \gg 1$. На границе такой среды с вакуумом при $y=0$ применимы граничные условия Леонтовича [6] для тангенциальных компонент электрических и магнитных полей

$$
[\mathbf{n}, \mathbf{E}]=-w[\mathbf{n},[\mathbf{n}, \mathbf{H}]]
$$

где $w=\sqrt{\mu / \varepsilon}-$ волновое сопротивление, $\mathbf{n}-$ вектор, направленный по нормали к поверхности.

Если в качестве материала рассматривается металл с высокой проводимостью на некоторой частоте $\omega$,

$$
\sigma \gg \omega \varepsilon^{\prime} / 4 \pi
$$

то можно приближенно считать $\varepsilon \approx-i \varepsilon^{\prime \prime}=-4 \pi \sigma i / \omega$. При этом выражение для волнового сопротивления принимает вид $w=-(1+i) k d / 2$, где $d=c(2 \pi \mu \omega \sigma)^{-1}-$ толщина скин-слоя.

При описании процесса усиления поле излучения на частоте $\omega$ в рамках исследуемой двумерной модели может быть представлено в виде волнового пучка ТМ-поляризации, имеющего следующие компоненты:

$$
\begin{gathered}
H_{x}=\operatorname{Re}\left(H_{x}^{\omega}(z, y) e^{i(\omega t-k z)}\right), E_{y}=\operatorname{Re}\left(E_{y}^{\omega}(z, y) e^{i(\omega t-k z)}\right), \\
E_{z}=\operatorname{Re}\left(E_{z}^{\omega}(z, y) e^{i(\omega t-k z)}\right),
\end{gathered}
$$

где $k=\omega / c$. Для монохроматических полей (3) граничные условия (1) эквивалентны введению эффективного поверхностного магнитного тока

$$
j_{x}^{m \omega}=\frac{c}{4 \pi} w H_{x}^{\omega} .
$$

С учетом магнитного тока и электрического тока компоненты полей связаны уравнениями Максвелла [7], которые могут быть приведены к виду

$$
\begin{gathered}
E_{z}^{\omega}=\frac{i}{k}\left(\frac{\partial H_{x}^{\omega}(z, y)}{\partial y}+\frac{4 \pi}{c} j_{z}^{e \omega} e^{i k z}\right), \\
E_{y}^{\omega}-\left(H_{x}^{\omega}(z, y)+\frac{i}{k} \frac{\partial H_{x}^{\omega}(z, y)}{\partial z}\right),
\end{gathered}
$$

$$
\frac{\partial E_{y}^{\omega}}{\partial z}-\frac{\partial E_{z}^{\omega}}{\partial y}=i k H_{x}^{\omega}+\frac{4 \pi}{c} j_{x}^{m \omega} \delta(y) .
$$

Здесь $\delta(y)$ - дельта функция, $j_{z}^{e \omega}-$ гармоника объемного электронного тока на частоте $\omega$, наводимого в пучке в результате развития резистивной неустойчивости.

В рамках квазиоптического подхода предположим, что амплитуда волнового пучка $H_{z}^{\omega}(z, y)-$ медленная функция координат в масштабе длины волны $\lambda=2 \pi / k$,

$$
\left|\frac{\partial H_{x}^{\omega}(z, y)}{\partial z}\right|, \quad\left|\frac{\partial H_{x}^{\omega}(z, y)}{\partial y}\right| \ll k\left|H_{x}^{\omega}(z, y)\right| .
$$

Это позволяет свести систему уравнений (5)-(7) к одному уравнению параболического типа для единственной компоненты магнитного поля [8]:

$$
-2 i k \frac{\partial H_{x}^{\omega}}{\partial z}+\frac{\partial^{2} H_{x}^{\omega}}{\partial y^{2}}=i \omega \frac{4 \pi}{c^{2}} j_{x}^{m \omega} \delta(y)-\frac{4 \pi}{c} \frac{\partial j_{z}^{e \omega}}{\partial y} e^{i k z} .
$$

Группировка частиц (модуляция плотности электронного пучка) происходит под действием продольной компоненты электрического поля (5) и в приближении относительно малого изменения энергии частиц $\left(\gamma-\gamma_{0}\right) / \gamma_{0} \ll 1$ описывается уравнением [8]:

$$
\frac{\partial^{2} \theta}{\partial z^{2}}=\frac{\tilde{\mu} e}{m c^{2} \gamma_{0}} \operatorname{Re}\left(i\left(\frac{\partial H_{x}^{\omega}}{\partial y}+\frac{4 \pi}{c} j_{z}^{e \omega} e^{i k z}\right) e^{\theta}\right),
$$

где $\tilde{\mu}=\gamma_{0}^{-2} \beta_{0}^{-3}-$ параметр инерционной группировки электронов, $\gamma$ - лоренц-фактор электрона, $\theta\left(y, z, \theta_{0}\right)=\omega t\left(y, z, \theta_{0}\right)-k z-$ его фаза в поле синхронной волны, $\theta_{0}=\omega t_{0}$ характеризует момент влета электрона в пространство взаимодействия.

Гармоника высокочастотного тока, наводимого в пучке в результате взаимодействия с электромагнитным полем, записывается как $j_{z}^{e \omega}=\pi^{-1} j_{z}^{e 0}(y) \int_{0}^{2 \pi} e^{-i \omega t} d \omega t_{0}$. Распределение тока представим в виде $j_{z}^{e 0}(y)=-I_{0} f(y) / b_{e}$, где $I_{0}$ - полный погонный ток, $f(y)$ - функция, описывающая невозмущенное распределение плотности электронов, $b_{e}=\int_{0}^{\infty} f(y) d y-$ эффективная толщина электронного потока.

Объединяя уравнение (9) для амплитуды квазиоптического волнового пучка и уравнения движения частиц (10), получим самосогласованную систему, описывающую электронно-волновое взаимодействие:

$$
\begin{gathered}
\frac{\partial H}{\partial Z}+i \frac{\partial^{2} H}{\partial Y^{2}}+i \chi \delta(Y) H=\frac{i}{B_{e}} \frac{\partial}{\partial Y}(F(Y) J), \\
\frac{\partial^{2} \theta}{\partial Z^{2}}=\operatorname{Re}\left(i \frac{\partial H}{\partial Y} e^{\theta}-\frac{i}{B_{e}}(F(Y) J) e^{i \theta}\right),
\end{gathered}
$$

где $J=j_{z}^{e \omega} e^{i k z} / j_{z}^{e 0}=\pi^{-1} \int_{0}^{2 \pi} \exp (-i \theta) d \theta_{0}-$ амплитуда высокочастотного электронного тока. Здесь введены следующие безразмерные величины: $Z=G k z$, 
$Y=\sqrt{2 G} k y, \quad B_{e}=\sqrt{2 G} k b_{e}, \quad H=\sqrt{2} e \mu H_{x}^{\omega} / m c^{2} \gamma_{0} k G^{3 / 2}$, $G=\left(2 \sqrt{2} \mu \lambda e I_{0} / m c^{3} \gamma_{0}\right)^{2 / 3}$ - параметр усиления (аналог параметра Пирса),

$$
\chi=i w / \sqrt{2 G}
$$

- нормированный комплексный импеданс, который при выполнении условия (2) можно представить в виде

$$
\chi=(1-i) \hat{w}, \quad \hat{w}=k d / 2 \sqrt{2 G} .
$$

В случае первоначально стационарного электронного пучка, равномерно распределенного по фазам влета при $Z=0$, граничные условия к уравнению (12) имеют вид

$$
\left.\theta\right|_{z=0}=\theta_{0} \in[0,2 \pi),\left.\quad \frac{\partial \theta}{\partial Z}\right|_{Z=0}=\Delta,
$$

где $\Delta=G^{-1}\left(\beta_{0}^{-1}-1\right)$. Предположим, что процесс усиления инициируется подачей входного сигнала достаточно малой величины в сечении $Z=0$ :

$$
\left.H(Y)\right|_{Z=0}=H_{0}(Y) .
$$

Из системы уравнений (11), (12) следует закон сохранения энергии

$$
\left.P\right|_{Z=L}-\left.P\right|_{Z=0}+P_{\text {ohm }}=4 \hat{\eta},
$$

где $P(Z)=\int_{0}^{\infty}|H(Z, Y)|^{2} d Y-$ интегральная мощность излучения. $P_{\text {ohm }}=2 \hat{w} \int_{0}^{L}|H(Z, Y=0)|^{2} d Z-$ нормированные омические потери. Электронный КПД дается соотношениями

$$
\begin{gathered}
\eta=\frac{G \hat{\eta}}{\tilde{\mu}\left(1-\gamma_{0}^{-1}\right)} \\
\hat{\eta}=\left.\frac{1}{2 \pi B_{e}} \int_{0}^{\infty} \int_{0}^{2 \pi}\left(\frac{\partial \theta}{\partial Z}-\Delta\right)\right|_{Z=L} F(Y) d \theta_{0} d Y,
\end{gathered}
$$

где $L=G k l_{z}$ - нормированная длина пространства взаимодействия.

Следует отметить, что самосогласованная система уравнений, аналогичная (11), (12), была исследована в [8] при анализе черенковского механизма усиления волн, распространяющихся над слабогофрированными поверхностями идеальных проводников. В этом случае поверхностный импеданс $\chi$ является действительной величиной, и соответственно в отсутствие электронного потока существуют собственные замедленные поверхностные волны, которые может возбуждать и усиливать прямолинейный электронный пучок.

В настоящей работе рассматривается случай, когда импеданс является существенно комплексной величиной. В условиях (2), когда импеданс задается соотношением (14), в отсутствие электронного потока решение уравнения (11) представляет собой поверхностную волну Ценнека [7]

$$
H \sim e^{-i \chi^{2} z}=e^{-2 \hat{w}^{2} z} .
$$

Эта волна в рамках квазиоптического приближения не является замедленной и не может быть возбуждена посредством черенковского механизма взаимодействия с прямолинейным электронным пучком. Соответственно развитие неустойчивости в такой системе обусловлено существованием в электронном потоке волн пространственного заряда, имеющих отрицательный знак энергии. При движении электронного пучка вблизи резистивной поверхности частичная диссипация энергии приводит к возбуждению таких волн.

\section{2. Линейная теория}

Рассмотрим далее модель, в которой электронный пучок однородной плотности и конечной ширины $B_{e}$ движется на расстоянии $B_{0}$ от безграничной по продольной координате импедансной поверхности. В приближении малого сигнала, линеаризуя систему уравнений (11), (12) и представляя амплитуды поля и высокочастотного тока в виде $H, J=\tilde{H}(Y), \tilde{J}(Y) \exp (-i \Gamma Z)$, где $\Gamma-$ нормированная комплексная поправка к продольному волновому числу $k$, получим дисперсионное уравнение, которое с точностью до определения значения импеданса совпадает с дисперсионным уравнением работы [8]:

$$
\begin{gathered}
\kappa[v-1]\left(1+\frac{1}{B_{e}(\Delta-\Gamma)^{2}-1}\right) \\
+g \frac{\left((g+\chi) e^{-g B_{0}}-(g-\chi) e^{g B_{0}}\right)}{\left((g+\chi) e^{-g B_{0}}+(g-\chi) e^{g B_{0}}\right)}[v+1]=0, \\
v=\frac{(\kappa-g)\left(B_{e}(\Delta-\Gamma)^{2}-1\right)+\kappa}{(\kappa+g)\left(B_{e}(\Delta-\Gamma)^{2}-1\right)+\kappa} e^{-2 \kappa B_{e}}, \\
g=\sqrt{\Gamma}, \quad k=\sqrt{\frac{\Gamma\left(B_{e}(\Delta-\Gamma)^{2}-1\right)}{B_{e}(\Delta-\Gamma)^{2}}}
\end{gathered}
$$

$i g, i \kappa-$ соответствующие комплексные поперечные волновые числа поля вне и внутри электронного потока, введенные по аналогии с обозначениями [8].

В случае предельно тонкого ленточного электронного слоя $\kappa B_{e} \ll 1$ можно приближенно записать $v \approx-1-2 \kappa B_{e}\left((\Delta-\Gamma)^{2} g^{-1}-1\right)$ и редуцировать дисперсионное уравнение (20) к виду

$$
\begin{aligned}
& \left(\Delta-g^{2}\right)^{2}(g-\chi)= \\
& \quad-\frac{g}{2} e^{-g B_{0}}\left((g+\chi) e^{-g B_{0}}-(g-\chi) e^{g B_{0}}\right) .
\end{aligned}
$$

При анализе дисперсионных уравнений (20) и (23) следует заметить, что поправка к продольному волновому числу и поперечные декременты спадания волн являются, вообще говоря, комплексными. Как показано 

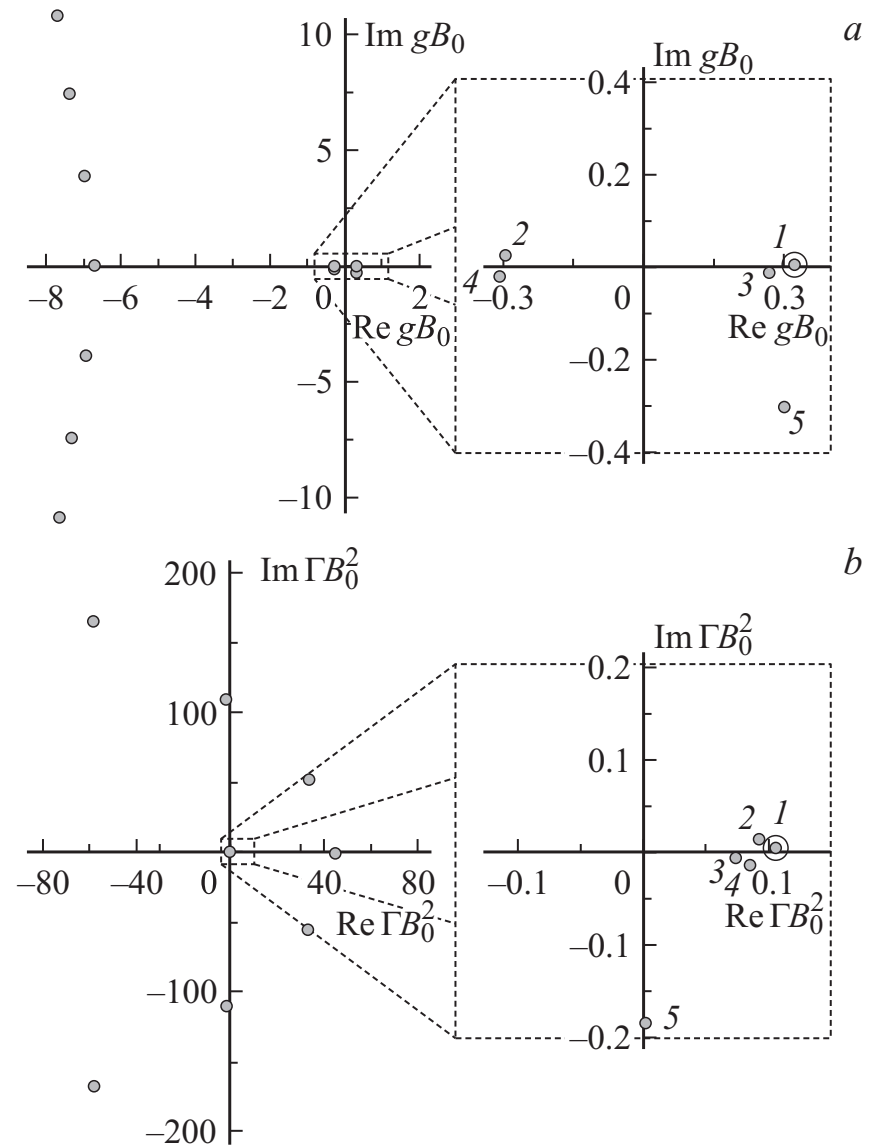

Рис. 2. Наименьшие по абсолютному значению корни уравнения (23). $a-$ нормированные поперечные волновые числа, $b-$ поправка к продольному волновому числу. Единственное решение, удовлетворяющее условиям (24), (25), соответствует индексу 1 на врезках с увеличенным масштабом; $\hat{w}=3, \Delta=9$, $B_{0}=0.1$.

в [8], с точки зрения процессов усиления излучения представляют интерес решения, удовлетворяющие следующим критериям:

$$
\operatorname{Re} g>0 \text { и } \operatorname{Im} g>0 .
$$

Такие решения соответствуют структуре электромагнитного поля, амплитуда которого спадает по оси у при удалении от пучка и импедансной поверхности и которое имеет поперечный поток энергии, направленный от пучка к периферии. Поскольку $\Gamma=g^{2}$, такая мода обладает положительным продольным инкрементом

$$
\operatorname{Im}(\Gamma)=2 \operatorname{Im}(g) \operatorname{Re}(g)>0 .
$$

В дальнейшем при анализе дисперсионных уравнений будем рассматривать в качестве поглощающего материала металл с большой проводимостью (2), когда поверхностный импеданс определяется соотношением (14). Ограничимся анализом случая тонкого ленточного пучка, в котором дисперсионное уравнение сводится к форме (23), содержащего три независимых действительных параметра: $\Delta, \hat{w}$ и $B_{0}$.
На рис. 2 представлены наименьшие по абсолютному значению корни уравнения (23) при $\hat{w}=3$, $\Delta=9, B_{0}=0.1$. Единственное решение, удовлетворяющее условиям (24), обозначено индексом „1“ на врезках с увеличенным масштабом. Это решение, фактически определяющее инкремент диссипативной неустойчивости, соответствует нормальной волне, нарастающей по продольной координате $Z$ и спадающей по поперечной координате $Y$. Все прочие корни по абсолютной величине превосходят представленные на рис. 2 и расположены в левой комплексной полуплоскости $\operatorname{Re} g<0$.

На рис. 3 для трех различных значений волнового сопротивления $\hat{w}$ приведены зависимости продольного инкремента от величины $\Delta$ при фиксированном зазоре между пучком и поверхностью $B_{0}=0.1$. На основании этих зависимостей можно сделать вывод о том, что для фиксированных значений $\hat{w}$ и зазора $B_{0}$ существует оптимальная скорость электронов $\vartheta_{0}$, однозначно связанная с оптимальной расстройкой $\Delta$, при которой инкремент резистивной неустойчивости достигает максимального значения.

Распределения амплитуд поперечной компоненты магнитного поля и действующей на электроны продольной компоненты электрического поля приведены на рис. 4. В случае ленточного электронного пучка и наводимого в нем продольного поверхностного электрического тока магнитное поле $H_{x}$ в соответствии с уравнениями Максвелла испытывает скачок, в то время как действующее на частицы продольное электрическое поле $E_{z}$ остается непрерывным. В этом смысле результаты аналогичны [8]. Вместе с тем существуют и заметные отличия. В случае резистивной неустойчивости по мере уменьшения абсолютного значения поверхностного импеданса или увеличения расстояния от поверхности до пучка $B_{0}$ распределение полей неустойчивой моды приближается к собственному полю электронного пучка, которое локализовано вблизи его поверхности, в то время как

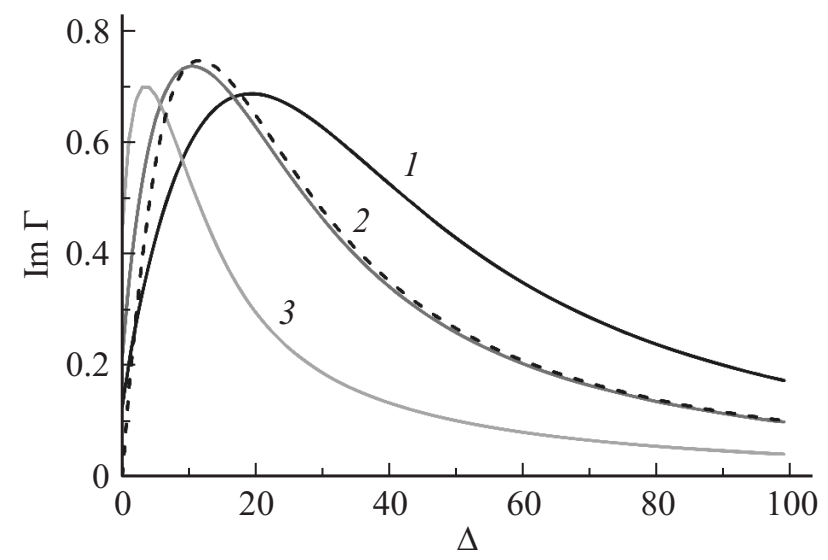

Рис. 3. Зависимости продольного инкремента $\operatorname{Im}(\Gamma)$ от величины $\Delta$ для различных значений импеданса: $1-\hat{w}=3$, $2-\hat{w}=2,3-\hat{w}=1$ и фиксированном зазоре между ленточным пучком и импедансной поверхностью $B_{0}=0.1$. Штриховая линия соответствует приближенному выражению (28) при $\hat{w}=2$. 

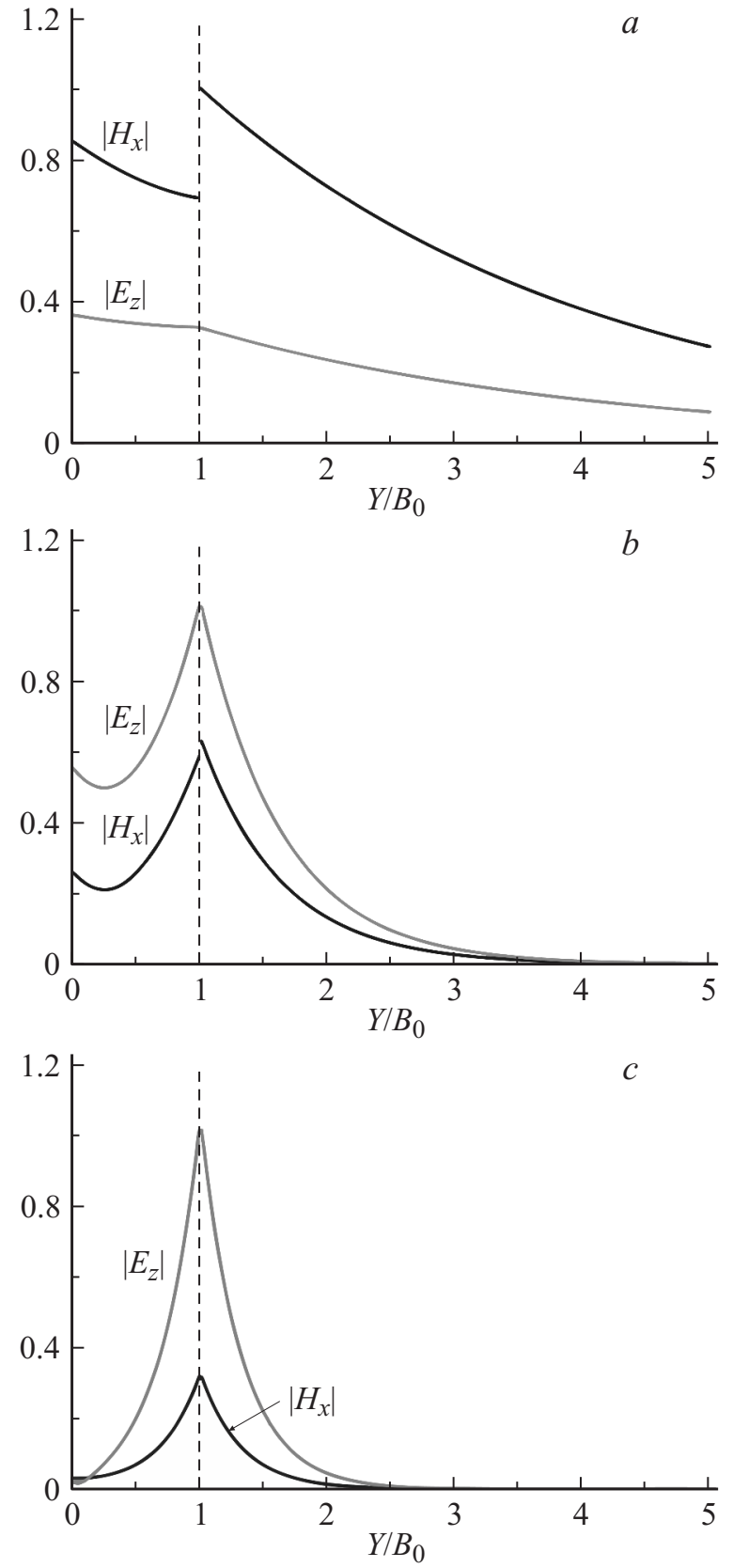

Pис. 4. Поперечные структуры модулей амплитуд магнитного поля $H_{x}$ и действующей на электроны продольной компоненты электрического поля $E_{z}$ для случая ленточного электронного пучка: $a-\hat{w}=3, \Delta=9, B_{0}=0.1 ; b-\hat{w}=3, \Delta=9$, $B_{0}=0.5, c-\hat{w}=0.5, \Delta=9, B_{0}=1$.

в случае рассмотренного в [8] чисто действительного импеданса усиливаемая волна остается близка к структуре замедленной „холодной“ моды, прижатой к гофрированной поверхности.

Уравнение (23) допускает приближенное аналитическое решение, в котором за основу берется пучковая волна движущегося в свободном пространстве периодически модулированного электронного пучка: при $\chi=0$ уравнение (23) имеет решение $g=\sqrt{\Delta}$, которое соответствует собственной пучковой волне. Поправка к продольному волновому числу, согласно (22), равна $\Gamma=g^{2}=\Delta$, а продольное волновое число $h$ дается формулой

$$
h=k(1+G \Gamma)=k\left(1+\frac{1-\beta}{\beta}\right)=\beta^{-1} k,
$$

что соответствует известной дисперсионной характеристике пучковых волн. В то же время поперечный декремент спадания полей пространственного заряда вне пучка, выраженный в физических переменных, в рамках представленного описания равен

$g_{\text {real }}=\sqrt{2 G} k \sqrt{\Delta}=k \sqrt{2\left(\beta_{0}^{-1}-1\right)} \approx \omega / \vartheta_{0} \gamma_{0}\left(1-\gamma_{0}^{-2} / 8\right)$,

что отличается от известной формулы $g_{\text {real }}=\omega / \vartheta_{0} \gamma_{0}$ на величину порядка $\gamma_{0}^{-2}$. Таким образом, использованное выше квазиоптическое приближение (пренебрежение второй производной по продольной координате $z$ в уравнении (9)) адекватно описывает структуру пучковых волн в области релятивистских энергий частиц $\gamma_{0} \gg 1$.

Как видно из рис. 2, при достаточно больших $\Delta$ корень уравнения (24), удовлетворяющий критериям (24), остается вблизи $\sqrt{\Delta}$. Соответственно решение $(23)$ при $\chi \neq 0$ можно искать в виде

$$
g=\sqrt{\Delta}-\xi, \quad \Gamma=g^{2} \approx \Delta-2 \sqrt{\Delta \xi},
$$

где $|\xi| \ll \sqrt{\Delta}$, после чего указанное дисперсионное уравнение редуцируется к уравнению второго порядка относительно малой поправки $\xi$ :

$$
\begin{aligned}
& \xi^{2}(\sqrt{\Delta}-(1-i) \hat{w})+\frac{1}{8 \sqrt{\Delta}} \\
& \times\left((\sqrt{\Delta}+(1-i) \hat{w}) e^{-2 \sqrt{\Delta} B_{0}}-(\sqrt{\Delta}-(1-i) \hat{w})\right)=0 .
\end{aligned}
$$

Уравнение (29) имеет два симметричных относительно $\xi=0$ решения. Корень с отрицательной реальной частью $\operatorname{Re} \xi<0$ соответствует быстрой волне пространственного заряда $\left(\vartheta_{p h}=\omega / h=c(1+\right.$ $+G(\Delta-2 \sqrt{\Delta} \operatorname{Re} \xi))^{-1}>\vartheta_{0}, \quad$ ср. с $\left.(26)\right)$, при этом соответствующая волна является затухающей по $z$, $\operatorname{Im} \Gamma \sim-\operatorname{Im} \xi<0$. В то же время корень с положительной реальной частью $\operatorname{Re} \xi>0$ соответствует медленной волне и имеет положительный инкремент $\operatorname{Im} \Gamma>0$ и, таким образом, удовлетворяет условиям (24). В результате для нормированного продольного инкремента резистивной неустойчивости получаем приближенное соотношение

$$
\operatorname{Im} \Gamma=2 \operatorname{Re} g \operatorname{Im} g=2(\sqrt{\Delta}-\operatorname{Re} \xi) \operatorname{Im} \xi \approx 2 \sqrt{\Delta} \operatorname{Im} \xi,
$$

где

$$
\xi=-\frac{1}{2 \sqrt{2} \Delta^{1 / 4}}\left(1-\frac{\sqrt{\Delta}+\hat{w}-i \hat{w}}{\sqrt{\Delta}-\hat{w}+i \hat{w}} e^{-2 \sqrt{\Delta} B_{0}}\right)^{1 / 2} .
$$


На рис. 3 штриховой линией представлена зависимость приближенного нормированного инкремента (30), (31) от энергии электронов $\Delta$ при фиксированных $\hat{w}=2$ и $B_{0}=0.1$. Видно, что в области максимальных инкрементов и при больших значениях параметра $\Delta=G^{-1}\left(\beta_{0}^{-1}-1\right)$ (где $G-$ малый параметр) формула (30) достаточно точно аппроксимирует решение уравнения (23). Различие проявляется лишь в области предельно больших энергий электронного пучка $($ малых $\Delta)$, где условие применимости $(28) \xi \ll \sqrt{\Delta}$ не выполняется.

Таким образом, как численное, так и приближенное аналитическое решение дисперсионного уравнения (23) показывает, что резистивная неустойчивость вблизи поглощающих поверхностей обусловлена неустойчивостью медленной волны пространственного заряда, имеющей отрицательный знак энергии [1] и нарастающей при наличии диссипации энергии в системе.

\section{3. Нелинейная стадия взаимодействия, оценки параметров}

Проведем здесь на основании уравнений (11),(12) анализ нелинейной стадии резистивной неустойчивости. Как следует из проведенного выше анализа собственных мод, поля излучения экспоненциально спадают по нормальной координате $y$. В этой связи для численного моделирования использовалось безотражательное граничное условие

$$
\left.\left(H+\frac{1}{\sqrt{\pi i}} \int_{0}^{Z} \frac{1}{\sqrt{Z-Z^{\prime}}} \frac{\partial H}{\partial Y} d Z^{\prime}\right)\right|_{Y=B}=0,
$$

которое ставилось в произвольном сечении $Y=B$, достаточно удаленном от источников.

В качестве первого примера рассмотрим резистивный усилитель на длину волны $3 \mathrm{~cm}$, в котором в качестве диссипативной среды выберем феррит. Такой материал использовался, в частности, в релятивистском клистроне [4], в пространстве дрейфа которого реализовалась резистивная неустойчивость. Следуя [4,9], будем считать проводимость $\sigma \approx 9 \cdot 10^{11} \mathrm{~s}^{-1}$ (в системе СИ $\sigma \approx 100 \mathrm{~S} / \mathrm{m})$, реальную часть диэлектрической проницаемости $\varepsilon^{\prime} \approx 10$ и магнитную проницаемость $\mu=1$. Соответственно в сантиметровом диапазоне выполнено условие (2), и при расчете электронно-волнового взаимодействия для оценки нормированного импеданса можно пользоваться приближенным выражением (14).

Параметры релятивистского электронного пучка возьмем близкими к рассмотренным в [4]: энергия частиц $\gamma_{0}=1.5(0.25 \mathrm{MeV})$, погонная плотность тока $1.3 \mathrm{kA} / \mathrm{cm}$, толщина пучка $2 b_{e}=1 \mathrm{~mm}$, расстояние до резистивной поверхности $b_{0}=0.5 \mathrm{~mm}$. Указанные физические параметры соответствуют следующим нормированным величинам: $G=0.6, B_{e}=0.2, B_{0}=0.1, \hat{w}=0.05, \Delta=0.57$. В результате по формуле (30) для линейной стадии

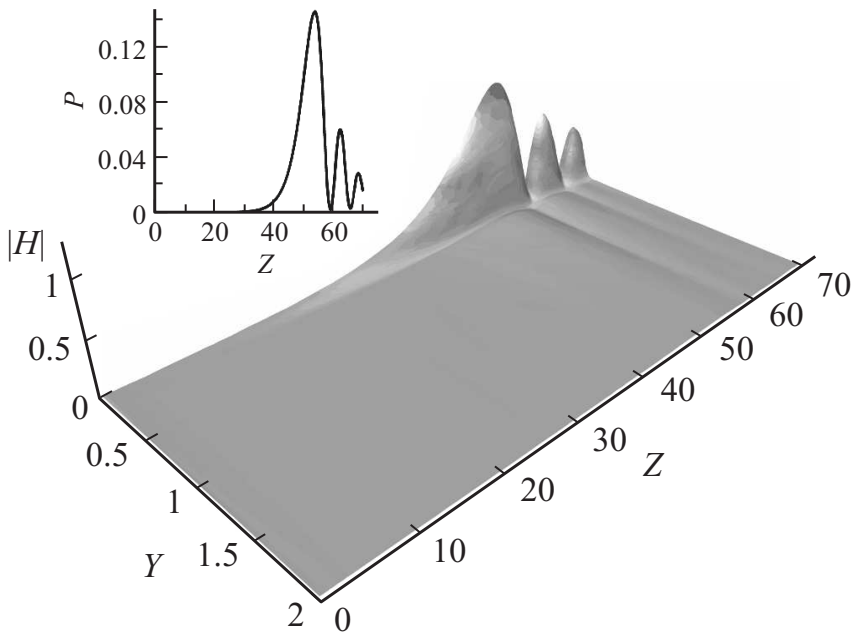

Рис. 5. Пространственное распределение модуля амплитуды усиливаемого волнового пучка и зависимость его мощности от продольной координаты: $G=0.6, B_{e}=0.2, B_{0}=0.1$, $\hat{w}=0.05, \Delta=0.57$.

усиления получим инкремент $0.1 \mathrm{~cm}^{-1}$, что близко к оценкам работы [4]. На рис. 5 приведены пространственное распределение амплитуды волнового пучка и зависимость интегральной мощности от продольной координаты для представленных выше нормированных параметров (на вставке). При моделировании нелинейной стадии развития диссипативной неустойчивости распределение плотности тока по поперечной координате задавалось в интервале $\left[b_{0}, b_{0}+2 b_{e}\right]$ функцией $f(y)=\sin ^{2}\left(\pi\left(y-b_{0}\right) / 2 b_{e}\right)$. Затравочный сигнал задавался как поле с постоянной на некотором отрезке по $y$ амплитудой и интегральной плотностью мощности $10 \mathrm{~kW} / \mathrm{cm}$.

Моделирование на основе уравнений (11),(12) показало, что выходная плотность мощности излучения в режиме насыщения равна $20 \mathrm{MW} / \mathrm{cm}$, что соответствует коэффициенту усиления по мощности $33 \mathrm{~dB}$. Насыщение усиления достигалось при нормированной длине $I_{z}=54$ или $l_{z}=43 \mathrm{~cm}$ в физических переменных. При достаточно высоком уровне усиления эффективность энергоотдачи электронного пучка была относительно низка: в рассматриваемом примере волновой КПД, т.е. отношение мощности излучения к мощности электронного потока, не превышал 6\%. Заметим, что электронный КПД, определяемый соотношением (18), составляет 56\%. Следовательно, значительная часть энергии электронного потока трансформировалась в омические потери.

В отличие от сантиметрового диапазона в коротковолновых диапазонах большие омические потери практически неизбежны при любом механизме взаимодействия. Например, в терагерцовых гиротронах в омические потери уходит до 90\% мощности, излучаемой электронным потоком [10]. Очевидным достоинством использования резистивного механизма является относительная простота системы в совокупности с ее компактностью. Та- 


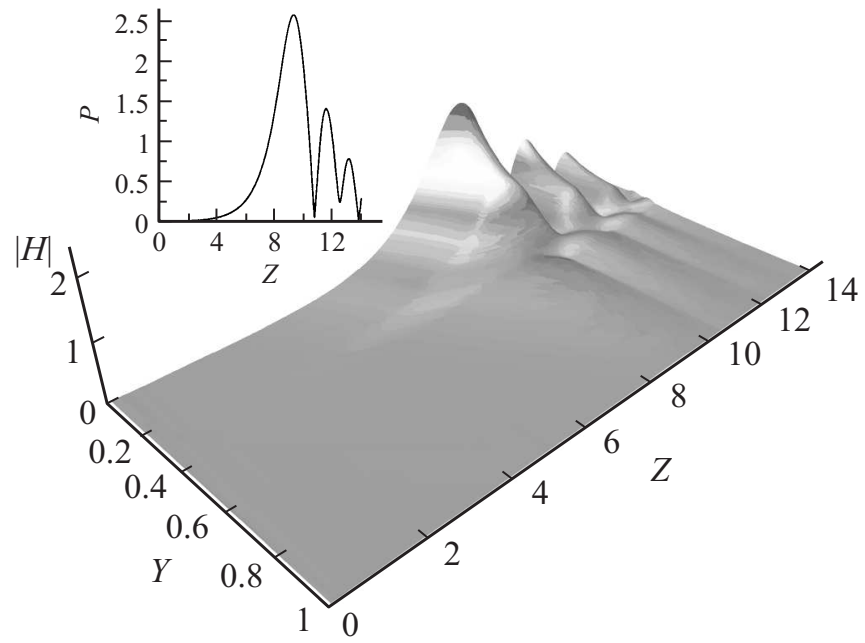

Рис. 6. Пространственное распределение модуля амплитуды усиливаемого волнового пучка и зависимость его мощности от продольной координаты: $G=4.3 \cdot 10^{-3}, B_{e}=0.2, B_{0}=0.2$, $\chi=3.35-0.3 i, \Delta=14$.

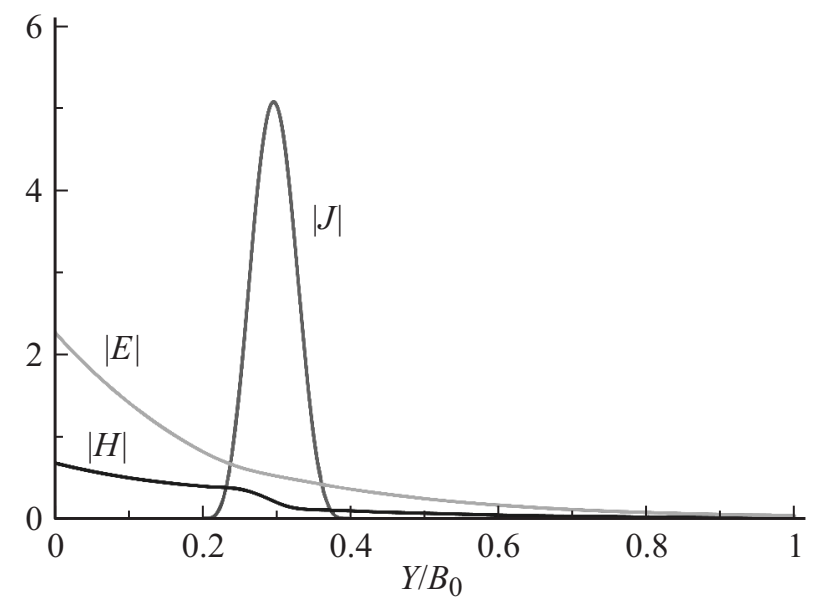

Рис. 7. Поперечные структуры нормированных амплитуд магнитного поля $H$, действующей на электроны продольной компоненты электрического поля $E=\partial H / \partial Y-i B_{e}^{-1} F(Y) J$ и высокочастотного тока $J$ на линейном этапе развития резистивной неустойчивости при нормированных параметрах, указанных на рис. 6.

ким образом, в коротковолновых диапазонах реализация источников, основанных на резистивной неустойчивости, представляется более актуальной.

Проведем моделирование диссипативного усилителя терагерцового диапазона на длину волны $\lambda=0.3 \mathrm{~mm}$. В этом диапазоне для феррита с указанными выше характеристиками условие (2) нарушается, и при записи волнового сопротивления вместо (14) следует использовать более общую формулу $\varepsilon=\varepsilon^{\prime}-4 \pi \sigma i / \omega$. Для оценок возьмем релятивистский электронный пучок со следующими параметрами: энергия частиц $1 \mathrm{MeV}$ $\left(\gamma_{0}=3\right)$, погонная плотность тока $1.3 \mathrm{kA} / \mathrm{cm}$, толщина электронного пучка $2 b_{e}=0.1 \mathrm{~mm}$, расстояние до ре- зистивной поверхности $b_{0}=0.1 \mathrm{~mm}$. Указанные физические параметры соответствуют нормированным величинам $G=4.3 \cdot 10^{-3}, B_{e}=0.2, B_{0}=0.2, \chi=3.35-0.3 i$, $\Delta=14$. Полученное в результате моделирования пространственное распределение медленной амплитуды магнитного поля представлено на рис. 6. Поперечные распределения медленных амплитуд полей и высокочастотного тока на линейной стадии развития диссипативной неустойчивости изображены на рис. 7. Выходная плотность мощности излучения в режиме насыщения равна $16 \mathrm{MW} / \mathrm{cm}$ при коэффициенте усиления по мощности $42 \mathrm{~dB}$ (интегральная плотность мощности затравочного сигнала $1 \mathrm{~kW} / \mathrm{cm})$. Насыщение усиления достигается на нормированной длине $L_{z}=9.3$ или $l_{z}=10.2 \mathrm{~cm}$. Волновой и электронный КПД составляют $1.2 \%$ и $6.3 \%$ соответственно, что существенно ниже значений, полученных в приведенном выше примере трехсантиметрового усилителя. Тем не менее абсолютное значение выходной мощности заведомо представляет практический интерес для терагерцового диапазона.

\section{Заключение}

Таким образом, на основе эффекта диссипативной неустойчивости, развивающейся при движении электронного потока над резистивной поверхностью, могут быть получены достаточно высокие коэффициенты усиления излучения коротковолновых, в частности терагерцового, диапазонов при относительно низкой эффективности энергообмена. Тем не менее абсолютные значения выходных мощностей в сочетании с компактностью электродинамической системы делают оправданным продолжение исследований усиления и генерации волн на основе исследуемого механизма. Важным вопросом, оставшимся за рамками настоящего анализа, является вопрос ввода и вывода излучения. В качестве одного из решений здесь может быть использование периодических брэгговских структур [11], для описания которых также может быть использован квазиоптический подход [8]. Заметим, что брэгговские структуры могут быть применены и в качестве частотно-селективных отражателей для реализации генераторных режимов на основе диссипативной неустойчивости.

Работа поддержана Российским фондом фундаментальных исследований, грант № 16-02-00890 А.

\section{Список литературы}

[1] Незлин М.В. // УФН. 1976. Т. 120. Вып. 3. С. 481-495.

[2] Лебедев А.Н., Шальнов А.В. Основы физики и техники ускорителей. М.: Энергоатомиздат, 1991. С. 271-278.

[3] Neil V.K., Sessler A.M. // Rev. Sci. Instrum. 1965. Vol. 36. C. 429.

[4] Han S. Uhm // Phys. Plasmas. 1994. Vol. 1. C. 2038.

[5] Ораевский А.Н., Сметанин И.В. // Письма в ЖЭТФ. 1995. т. 62. Вып. 3. С. 242-246. 
[6] Леонтович М.А. // Изв. АН СССР. Сер. физ. 1944. Т. 8. Вып. 1. С. 16-21.

[7] Вайнштейн Л.А. Электромагнитные волны. М.: Радио и связь, 1988. 440 с.

[8] Гинзбург Н.С., Малкин А.М., Железнов И.В., Сергеев А.С., Кочаровская Е.Р. // ЖТФ. 2016. Т. 86. Вып. 11. С. 6.

[9] Smit J., Wijn H.P.J. // Ferrites, Philips' Technical Library, 1959.

[10] Glyavin M.Yu,. Luchinin A.G., Golubiatnikov G.Yu. // Phys. Rev. Lett. 2008. Vol. 100. P. 015101.

[11] Климов В.В. Наноплазмоника. М.: Физматлит, 2009. 480 с. 